Editorial, Molecular and Cellular Neuroscience SI on Biomarkers for Neurodegenerative Diseases

\title{
Disease signatures: biomarkers/indicators of neurodegeneration
}

Henrik Zetterberg ${ }^{1,2,3,4}$ and Mathias Bähr ${ }^{5}$

${ }^{1}$ Clinical Neurochemistry Laboratory, Sahlgrenska University Hospital, S-431 80 Mölndal,

Sweden

${ }^{2}$ Department of Psychiatry and Neurochemistry, Institute of Neuroscience and Physiology, the Sahlgrenska Academy at the University of Gothenburg, S-431 80 Mölndal, Sweden

${ }^{3} U K$ Dementia Research Institute at UCL, London WC1E 6BT, UK

${ }^{4}$ UCL Institute of Neurology, Department of Neurodegenerative Disease, Queen Square, London WC1N 3BG, UK

${ }^{5}$ University Medical Center, Department of Neurology, Robert-Koch Strasse 40, 37075

Goettingen, Germany

Correspondence:

Henrik Zetterberg, MD, PhD

Institute of Neuroscience and Physiology

Department of Psychiatry and Neurochemistry

The Sahlgrenska Academy at the University of Gothenburg

S-431 80 Mölndal

\section{SWEDEN}

Tel (office): +46 313430142

Tel (cell): +46 768672647

Tel (secretary): +46 313430025

Fax: +46 31419289

E-mail: henrik.zetterberg@gu.se 
A biomarker may be defined as a distinct characteristic that is measured as an indicator of a normal biological process, a pathogenic process, or a response to an exposure or therapeutic intervention. They have become ubiquitous across neurodegenerative disease research and increasingly influence clinical diagnosis-making and care. For most of the neurodegenerative diseases, there is a biologic diagnosis gold standard (autopsy) distinct from the clinical diagnosis (symptom-based cognitive and functional criteria). Diagnostic biomarkers for neurodegenerative diseases are aimed at detecting the former, irrespective of the clinical state of the patient. Clinically, biomarker data on potential underlying pathologies have to be carefully evaluated, together with the medical history and clinical signs of the patient, to sort out what pathologies are most likely to contribute to the patient's symptoms. This is a challenging process that hopefully will translate into better care, and, in the future, new treatments that can be prescribed in a personalized manner.

In Alzheimer's disease (AD), combining longitudinal cohorts with robust biomarkers validated against neuropathology has led to major advances in our understanding of the pathological sequence that underpins AD (Veitch et al., 2019). Accumulation of amyloid $\beta$ $(\mathrm{A} \beta)$ in the brain is a very early event, starting a decade or two before symptoms become apparent. $A \beta$ can measured using two broadly interchangeable biomarkers: cerebrospinal fluid (CSF) A $\beta 42 / A \beta 40$ ratio and amyloid positron emission tomography (PET) (Blennow et al., 2015). Recently, the CSF $A \beta 42 / A \beta 40$ ratio has been translated into a promising blood test that may find utility for screening in primary care and clinical trials (Zetterberg, 2019). In parallel with biomarker evidence of $\mathrm{A} \beta$ accumulation, CSF concentrations of total and phosphorylated tau increase (Fagan et al., 2014), likely indicating an A $\beta$-related change in tau metabolism resulting in increased secretion of tau proteins from affected neurons. This tau dysfunction eventually manifests itself as tangle pathology and neurodegeneration, which can be visualized using tau PET imaging and magnetic resonance imaging, respectively (Okamura et al., 2018; Weston et al., 2015), and correlate more closely with cognitive decline. A $\beta$ and tau biomarkers have recently been incorporated into diagnostic criteria in which biomarker data may support a diagnosis of AD in its preclinical, mild cognitive impairment or dementia stage, according to symptom-based cognitive and functional criteria (Dubois et al., 2016).

Whilst there are well-validated biomarkers for A $\beta$ and AD-type tau pathologies, we currently lack robust biomarkers for a number of other neurodegenerative pathologies, including nonAD-type tau, TDP-43 and $\alpha$-synuclein inclusions. However, intense research is ongoing to 
develop such biomarkers. Another very intense research topic is to translate some of the biomarker candidates that require advanced neuroimaging or CSF sampling into more accessible blood tests.

This special issue of Molecular and Cellular Neuroscience is aimed at covering all of these topics. The first two articles give an updated account of imaging and fluid biomarkers for A $\beta$ and tau pathologies (Cohen et al., 2018; Scholl et al., 2018). The following paper covers biomarkers for synaptic dysfunction and degeneration (Heurling et al., 2019). The forth paper deals with biomarker candidates for TDP-43 pathology; they are currently elusive but progress is being made (Steinacker et al., 2018). The last three contributions summarize recent developments in biomarkers for Parkinson's disease, Huntington's disease and prion diseases (Maass et al., 2018; Thompson and Mead, 2018; Zeun et al., 2019).

The biomarker field is rapidly evolving and we hope this special issue will serve as a springboard for further discussions and research on how to identify and validate additional biomarkers for neurodegenerative pathologies. If such projects are successful, we may see future in which a precision medicine approach may allow multiple biomarkers to show which pathologies are present in a certain patient seeking medical advice in clinical practice or being evaluated for potential enrolment in a clinical trial, and how novel drug candidates may influence such pathologies. This should translate into better care and treatment for our patients.

\section{Acknowledgements}

$\mathrm{HZ}$ is a Wallenberg Academy Fellow supported by grants from the Swedish Research Council (\#2018-02532), the European Research Council (\#681712), Swedish State Support for Clinical Research (\#ALFGBG-720931) and the UK Dementia Research Institute at UCL.

\section{References}

Blennow, K., Mattsson, N., Scholl, M., Hansson, O., and Zetterberg, H. (2015). Amyloid biomarkers in Alzheimer's disease. Trends Pharmacol Sci 36, 297-309.

Cohen, A.D., Landau, S.M., Snitz, B.E., Klunk, W.E., Blennow, K., and Zetterberg, H. (2018). Fluid and PET biomarkers for amyloid pathology in Alzheimer's disease. Mol Cell Neurosci. 
Dubois, B., Hampel, H., Feldman, H.H., Scheltens, P., Aisen, P., Andrieu, S., Bakardjian, H., Benali, H., Bertram, L., Blennow, K., et al. (2016). Preclinical Alzheimer's disease:

Definition, natural history, and diagnostic criteria. Alzheimers Dement 12, 292-323.

Fagan, A.M., Xiong, C., Jasielec, M.S., Bateman, R.J., Goate, A.M., Benzinger, T.L., Ghetti, B., Martins, R.N., Masters, C.L., Mayeux, R., et al. (2014). Longitudinal change in CSF biomarkers in autosomal-dominant Alzheimer's disease. Sci Transl Med 6, 226ra230.

Heurling, K., Ashton, N.J., Leuzy, A., Zimmer, E.R., Blennow, K., Zetterberg, H., Eriksson, J., Lubberink, M., and Scholl, M. (2019). Synaptic vesicle protein 2A as a potential biomarker in synaptopathies. Mol Cell Neurosci.

Maass, F., Schulz, I., Lingor, P., Mollenhauer, B., and Bahr, M. (2018). Cerebrospinal fluid biomarker for Parkinson's disease: An overview. Mol Cell Neurosci.

Okamura, N., Harada, R., Ishiki, A., Kikuchi, A., Nakamura, T., and Kudo, Y. (2018). The development and validation of tau PET tracers: current status and future directions. Clin Transl Imaging 6, 305-316.

Scholl, M., Maass, A., Mattsson, N., Ashton, N.J., Blennow, K., Zetterberg, H., and Jagust, W. (2018). Biomarkers for tau pathology. Mol Cell Neurosci.

Steinacker, P., Barschke, P., and Otto, M. (2018). Biomarkers for diseases with TDP-43 pathology. Mol Cell Neurosci.

Thompson, A.G.B., and Mead, S.H. (2018). Review: Fluid biomarkers in the human prion diseases. Mol Cell Neurosci.

Veitch, D.P., Weiner, M.W., Aisen, P.S., Beckett, L.A., Cairns, N.J., Green, R.C., Harvey, D., Jack, C.R., Jr., Jagust, W., Morris, J.C., et al. (2019). Understanding disease progression and improving Alzheimer's disease clinical trials: Recent highlights from the Alzheimer's Disease Neuroimaging Initiative. Alzheimers Dement 15, 106-152.

Weston, P.S., Simpson, I.J., Ryan, N.S., Ourselin, S., and Fox, N.C. (2015). Diffusion imaging changes in grey matter in Alzheimer's disease: a potential marker of early neurodegeneration. Alzheimers Res Ther 7, 47.

Zetterberg, H. (2019). Blood-based biomarkers for Alzheimer's disease-An update. J Neurosci Methods 319, 2-6.

Zeun, P., Scahill, R.I., Tabrizi, S.J., and Wild, E.J. (2019). Fluid and imaging biomarkers for Huntington's disease. Mol Cell Neurosci. 